\title{
MORPHOMETRY AND LASER SCANNER IMAGING: A REVOLUTION IN ANATOMY
}

\section{Giacomo Gelati, Mario Tanga}

\section{Corpus (International Group for the Cultural Studies of the body)}

\begin{abstract}
Anatomy is a visual science, even if the visual quality of anatomy can be declined in different ways, as we will show. Anatomy holds its own main feature in the etymology of the name itself: it is through cutting ( $\alpha v \alpha$, anà) by lancet ( $\tau \dot{\varepsilon} \mu v \omega$, tèmno) that preparations are yet prepared. This operation is aimed to make evident and well visible anatomical structures. Vesalius is the first modern anatomist and his main heuristic principle consists in the equivalence between seeing and knowing. The possibility of analyzing fresh anatomical preparations is made short by the unavoidable decomposition processes of the cadaver. Due to this reason beautiful and precious tables have always been drawn, painted and printed. We remember the ones by the Fisiocritic Paolo Mascagni, whose double centenary of death is celebrated this year. Watercolor tables are yet much realized and used. However they are bi-dimensional schematizations and, even if well done, they remain far from reality. Photography allows to fix the image of anatomical preparations with high fidelity of particulars. However these images are static. The graphic synthesis allows to realize four-dimensional human virtual models. They can be rotated according to the three spatial axes and, thanks to this, they can be observed from every point of view. Due to the fact that these are schematizations, they are very far from reality. For this reason CT three-dimensional reconstruction, that rebuilds anatomy in three dimensions, allows to obtain results with very superior quality and fidelity. However these reproductions lack color and real light. The gap between the iconographic representation and the existing thing is and will always be not fully eliminable. However our use of laser scanner technology allows to reduce this gap to minimal levels, with a quick and easy acquisition process. Laser scanner generates a cloud of points of the examined object. Each point is identified through exact coordinates. Besides, the photos of the same object can be over-placed to the cloud. The result is a virtual model that reconstructs the real object, highly corresponding as in morphology and as in colors. This virtual model allows us to interact and we can rotate it, watch at it from every perspective and especially we can measure it. The scanner we have used allowed us to reach an accuracy of $\pm 25 \mu \mathrm{m}$. The anatomical preparation is literally "immortalized", up to under-millimetric details, where the naked eye is ineffective. The so obtained image allows to re-observe and to measure the object forever. We can imagine a lot of very innovative, if not revolutionary applications. We realized our four-dimensional models aiming to attach them to this project. These scanning are of two skulls and of a heart. They are the concrete proof of the possibility of obtaining surprising results in many areas, from normal anatomy to pathological anatomy, from legal medicine to biology. This way of obtaining anatomical images is marking a turning point from a taxonomic, serial and verbal conception of Anatomy to a visual, spatial and mathematical one. Instead of lists of nominal labels we have now coordinates and quantitative/structural references. This makes Anatomy more treatable through digital methods. The visual approach has far origins: since XIII Century, when real (not formal) Renaissance of figurative arts begins, and during following ages, visual paradigms gain more and more importance in human knowledge. Once more we are dwarves on the shoulders of giants. Besides, detecting morphology by laser scanner pushes us to re-configure the relationship between nomothetic and ideographic approach in building scientific models.
\end{abstract}

Key words: Anatomy; atlas; biology; immersive anatomy; laser scanner; legal medicine; morphometry.

\section{INTRODUCTION}

Anatomy is a visual science that holds its own main feature in the etymology of the name itself: it is through ( $\alpha v \alpha$, anà) cutting by lancet ( $\tau \varepsilon ́ \mu v \omega$, tèmno) that preparations are yet prepared. This operation is aimed to make evident and well visible anatomical structures. Andreas Vesalius is the first modern anatomist and his main heuristic principle consists in the equivalence between seeing and knowing.
Nothing is as effective as the execution of a dissection; this is a big truth that will never fade. However, since ancient times, anatomists had to deal with the unavoidable decomposition processes of the cadavers, which make short the possibility of analyzing fresh anatomical preparations. For this reason beautiful and precious tables have always been drawn, painted and printed. We remember the ones by Leonardo da Vinci, by Andreas Vesalius and by the Fisiocritic Paolo Mascagni, whose double centenary of death is celebrated this year. Water-

Correspondence to:

Mario Tanga,

Corpus (International Group for the Cultural Studies of the Body)- http://corpus.comlu.com

E-mail:m.tanga@tin.it 
color tables are yet much realized and used, however they are bi-dimensional schematizations. Even if well done, they remain far from the existing object, since they are different from reality both under the epistemological and the cognitive point of view. We have always to remember that only the anatomical structures portrayed by the author (and in the way they have been portrayed by the author) are observable in the paintings of a common watercolor atlas.

Photography allows to fix the image of anatomical preparations with high fidelity of particulars, bypassing the artistic representation by the author. Even if photos can be layered and arranged in sequence, they remain static, so they offer a limited possibility of interaction with the observer.

The advent of the IT and of the graphic synthesis has allowed to realize four-dimensional human virtual models. They can be rotated according to the three spatial axes and, thanks to this, they can be observed from every point of view. They allow to visualize the whole body or to stress the particular structures we are interested in: we can zoom in on muscles, nerves, vessels, on their topographic relation, we can observe the organs in situ or isolated. It is possible to observe cavities, organs or the whole body dissected according to sagittal, frontal or transverse planes. It is also given the possibility of resecting superficial anatomical structures, in order to reach and to make evident deeper planes and vice versa. For sure the possibility of observing the examined object both intact and dissected and from every point of view, according to the rotation perspective, represents a big help. However, since these are schematizations, they are very far from reality, apart from the accuracy. For this reason CT three-dimensional reconstruction, that rebuilds Anatomy in three dimensions, allows to obtain results with very superior quality and fidelity. It is fundamental to emphasize the fact that these reproductions lack color and real light.

The gap between the iconographic representation and the existing thing is and will always be not fully eliminable. However our use of laser scanner technology allows to reduce this gap to minimal levels, with a quick and easy acquisition process. Laser scanner generates a cloud of points of the examined object. Each point is identified through exact coordinates. Besides, the photos of the same object can be layered to the cloud. The result is a virtual model that reconstructs the real object, highly corresponding as in morphology and as in colors. This virtual model allows us to interact and we can rotate it, watch at it from every perspective and especially we can measure it. The laser scanner was born as a measuring instrument in industry and it maintains such feature in this new field, allowing to quantify with high precision the area of the organs, the diameters, the volumes and so on. The EDGE ScanArm $\mathrm{HD}{ }^{\circledR}$ we have used allowed us to reach an accuracy of $\pm 25 \mu \mathrm{m}$. The anatomical preparation is literally "immortalized", up to under-millimetric details, where the naked eye is ineffective. The so obtained image allows to re-observe and to measure the object forever.

We can imagine a lot of very innovative, if not revolutionary applications. We realized our four-dimensional models aiming to attach them to this project. These scanning are of two skulls and of a heart and they are the concrete proof of the possibility of obtaining surprising results in many areas, from Normal Anatomy to Pathological Anatomy, from Legal Medicine to Biology.

This way of obtaining anatomical images is marking a turning point from a taxonomic, serial and verbal conception of Anatomy to a visual, spatial and mathematical one. Instead of lists of nominal labels we have now coordinates and quantitative/structural references. This makes Anatomy more treatable through digital methods. The visual approach has far origins: since XIII Century, when real (not formal) Renaissance of figurative arts begins, and during following ages, visual paradigms gain more and more importance in human knowledge. Once more we are dwarves on the shoulders of giants.

Besides, detecting morphology by Laser Scanner pushes us to re-configure the relationship between nomothetic and ideographic approach in building scientific models.

\section{APPLICATIONS IN NORMAL ANATOMY AND IN PATHOLOGICAL ANATOMY}

For physicians, non-medical staff and for any researcher in the field of body studies, Human Anatomy represents the most part of the basis of the whole knowledge and it reveals to be the key element for the comprehension of many circumstances, as in the period of the studies, as in the profession. For this reason an accurate and organic background is an absolute duty. In the phase of the university education the dissection of a fresh cadaver represents the most effective and preferable approach, however the use of an atlas cannot be renounced during the "domestic" study on the anatomical treatise. We have to remember that fresh preparations are not always easily accessible (because of many reasons: strict law code, religion, small number of body donors, etc.) and that they can be analysed only for a short period of time.

The laser scanner is the perfect instrument to be employed in the realization of a completely new Human Anatomy Atlas (and not necessarily only human...) no more based on static tables, but on a four-dimensional model, that is to say a three-dimensional model which can be rotated and observed from every perspective, with an absolute fidelity to reality, closely linked to the existing object and analyzable as in a real dissection. Many scanning, realized in different moments, can be layered and processed with a specific software, allowing 
to obtain the same features of the virtual atlases formerly described, with the new possibility of measuring the anatomical model with high precision and authenticity. Moreover this will be the first Human Anatomy Atlas in which the anatomical preparation is inserted in a virtual sphere, that represents a reference system made up of meridians and parallels. This will make the virtual handling of the model itself easier and more accurate.

It is important to stress a key point. In this case the observer has the opportunity of analyzing no more a schematization, but a model made through an objective instrumental data acquisition process, highly corresponding in colors, in light and in morphology, from the macroscopic aspect to the under-millimetric details, where the naked eye is ineffective, visible only after enlargement. Video clips from laparoscopic or robotic surgical operations may be attached to the atlas and thanks to this multimedia equipment it would be possible to visualize the anatomical planes while the surgeon is moving across them. This approach would be very helpful in the comprehension of the way the morphogenesis shapes the topographic relations between the organs.

Thanks to a specific software it is possible to convert the 3D textured model so that it can be watched with 3D glasses. In this way the anatomical preparation literally gets out of the monitor screen and matches the observer, maintaining a very high resolution which is fully noticeable only after strong enlargement: this is an experience that for medical staff, students, scholars, health-care workers and science lovers is really useful from a practical point of view, but also exciting and without precedent.

In the end a set of digital histological slides may be attached to the atlas and maybe a set of radiological images too. A so conceived and realized product has the highest completeness and it represents the perfect instrument to study Anatomy in an organic, accurate and detailed way.

This technology and the way of application we have described can be employed both in normal anatomy, as in pathological anatomy. Every day new clinical cases, characterized by new pathological features, or by novelty elements of already known pathologies, are published in literature, showing the problem from all the different perspectives: from the biochemical point of view, to the histopathological one, from the clinic features to the Anatomical aspects revealed by autopsy. Laser scanner data acquisition gives the possibility of producing a completely new Pathological Anatomy Atlas and thanks to the quickness and easiness of the scanning process itself, it would be very simple to update the literature iconography, despite the billions of coordinates that are at the basis of the models: very high quality in a very simple way.

This completely new approach represents the beginning of the 3.0 anatomical imaging era, which is preceded by 1.0 era of watercolor tables, by 1.2 era of photographic atlases and by 2.0 era of virtual graphic synthesis three-dimensional pictures.
Moreover, [for the first time] anatomy is hit by the so called "data deluge" and enters the Big Data Age. There is a very big dimensional/informational gap between the world of Laser Scanning 3D Models and the world of watercolor tables, of photos and of virtual graphic synthesis three-dimensional models. The digital anatomical preparations, obtained with laser scanning techniques, can be observed and measured with an accuracy of \pm 25 $\mu \mathrm{m}$, that is to say with a resolution which is much higher than human eye's potentialities. As a consequence, the informational content of these pictures is beyond the physiological limits of our naked eye: only after enlargement the under-millimetric structures become analyzable. This is made possible by the billions of points identified by specific coordinates- the models are made of, which can be processed and managed only through the powerful computers existing today.

Each point and all points are not a bare offer of viewable points, surfaces or volumes: each one contains the quantitative information about its position in the structure, exactly matching with the real object. So our operating on the model (measuring 1/2/3 dimensional extensions, etc.) can replace the operating on reality, but in an easier and more warranted way. Besides, our operation can be directly saved and treated digitally.

Let us cast our mind into the future, maybe into a not very distant future, and let us imagine how one day Anatomy will be studied and taught, with high probability. When the technology of 3D holographic projectors will be developed and will be within everybody's reach, the same scanning that today can be observed on a screen will be projected in the three-dimensional space, giving to every student the possibility of executing and of observing virtual dissections of real bodies, at home or in any other place. The Human Atlas we have talked about will become literally three-dimensional and four-dimensional. In the lecture halls the dissections of the cadavers will be accompanied by the projection of 3D holographic models of real organs at high enlargement and maybe, through the use of a powerful 3D holographic projector, students will walk inside body cavities, such as the thoracic cavity, or the abdominal cavity, they will explore on foot the cranial fossae and they will walk inside the lumen of hollow organs. Anatomy will become immersive.

\section{APPLICATIONS IN LEGAL MEDICINE}

The laser scanner, thanks to its unique and inimitable capacity, can survey the least particulars of the analyzed objects and it can assign specific coordinates to each point. Consequently it gives the possibility of measuring with high precision lengths, angles, areas or volumes even after days, months, years and, virtually, forever. It is very important to underline that these three-dimensional models are the product of an objec- 
tive instrumental data acquisition process, not falsifiable, absolutely accurate in morphology, both in the macroscopic aspect as in the under-millimetric details.

Such morphologic and morphometric precision and the possibility of analyzing without any limit of time these objective instrumental data, match the requirements of legal medicine in a perfect way.

At this point we introduce another laser scanner model that we have used in the realization of this project: the DPI-8 Handheld Scanner ${ }^{\circledR}$. This instrument is analogous to the EDGE ScanArm HD ${ }^{\circledR}$ that we have already showed, but it has a lower accuracy, suitable for the different range and for the different use. It is characterized by small dimensions, quick data acquisition capacity, simplicity in use and in transport. This scanner, since it has an integrated camera, is able to generate the 3D model from the cloud of points and to layer the photographic texture in real time. It is the perfect instrument to survey a crime scene, to scan and to photograph at the same time wide spaces such as rooms or outdoors areas. Each object in the field is "immortalized" in its parts and reciprocal positions with the environment. We have attached to this project the survey of a table and of a heart lying on it, realized with the DPI-8 Handheld Scanner ${ }^{\circledR}$ in order to show the way this instrument works and the offered potentialities.

When we focus the attention on the details of a cadaver or on any other object whose dimensions are in the order of magnitude of $10^{-2} \mathrm{~m}$, a higher level of accuracy is required and the use of the EDGE ScanArm HD ${ }^{\circledR}$ becomes essential. However both instruments are fundamental since they are complementary and they work synergetically: the choice for the use of one the two depends on the needs of the operator in accuracy and range.

The revolutionary element consists in the fact that a survey realized with this technology represents an absolutely objective instrumental source of data, which can be analyzed without any limit of time. Consequently everything can be reviewed. No detail is missed by laser scanner, thanks to which it is possible to eliminate every kind of human error and omission in surveying and the changes of the examined object in time: from the decomposition processes of a cadaver, to the changes in a crime scene, to the tampering with evidence and so on.

\section{APPLICATIONS IN BIOLOGY}

On this point we mention as an example of application another project of ours, introduced together with this one at the Academy of the Fisiocritici and to be published soon: "Possible Contributions For The Development of An Exaptive Theory: The Example Of Rachis". The morphologic and morphometric analysis of vertebras, realized through laser scanner technology, allowed to reach a comparative valuation of rachidian exaptive transforma- tions in different typologies of animals: chamois, common fin whale, bottlenose dolphin and Man.

\section{ACKNOWLEDGEMENTS}

We would like to thank our President Doctor Sara Ferri, Doctor Giuseppe Manganelli, Doctor Fabrizio Cancelli and the whole Accademia dei Fisiocritici for the wide availability and the precious collaboration.

We would like also to thank Massimo Piazzesi, Donato Marcantonio and Matteo Lapini of Spektra S.r.l. and Nadia Guardini, Alberto Brodi and Nicola Sorrentino of MeSa S.r.l. who have offered the instruments and the technological support for the realization of this project.

\section{BIBLIOGRAPHY}

Alcañiz M, Grau V, Monserrat $C$, et al. A system for simulation and planning of orthodontic treatment using a low cost $3 D$ laser scanner for dental anatomy capturing. In: JD Westwood, HM Hoffman, RA Robb, D Stredney (eds). Medicine Meets Virtual Reality. Amsterdam: IOS Press; 1999. pp 8-14.

Aung SC. Evaluation of the laser scanner as a surface measuring tool and its accuracy compared with direct facial anthropometric measurements. Br J Plast Surg 1995:48:551-8.

Baroni G, Patete P, Riboldi M, Spadea MF. Hand-held laser scanner for 4-D anatomical surface modeling. 2007. Available from: http://hdl.handle.net/11311/245248.

Fraccaro V. Un diluvio di dati. In: Le Scienze, n. 568, Dicembre 2015, pp. $70-77$

Hulse M, Tam M, Isherwood S, et al. Production of 3-D printer-generated radiotherapy treatment shells using DICOM CT, MRI or 3-D surface laser scan-Acquired STL files: preclinical feasibility studies. $8^{\text {th }}$ NCRI Cancer Conference, 2-5 November 2014, Liverpool, UK.

Leondes CT (ed). Medical imaging. Systems techniques and applications. Amsterdam: Gordon and Breach Sciences Publishers; 1997.

McMenamin P. Quayle MR, McHenry CR, Adams JW. The production of anatomical teaching resources using the three-dimensional (3D) printing technology. Am Assoc Anatom 2014;7:479-86.

SmartGeoMetrics. Mapping anatomy with laser scanners benefits researchers and video game developers. 2014. Available from: http://www.smartgeometrics.com/blog/video-gamedevelopment/mapping-anatomy-with-laser-scanners-benefits-researchers-and-video-game-developers/

Tognola G, Parazzini M, Ravazzini P, Svelato LC. Simple 3D laser scanner for anatomical parts and image reconstruction from unorganized range data. IN: IEEE Instrumentation and Measurement Technology Conference, vol.1, pp 171 -174. February 2002.

Vegetti M. Il coltello e lo stilo. Milano: Il Saggiatore; 1996.

Villa C, Gaudio D, Cattaneo C, et al. Surface curvature of pelvic joints from three laser scanners: separating anatomy from measurement error. J Forensic Sci 2015;60:374-81.

Welsh E, Anderson P, Rea P. A novel method of anatomical data acquisition using the Perception Scan Works V5 Scanner. Available from: http://www.ijlsm.org/download/1415244503.pdf 